\title{
(2) OPEN ACCESS \\ Prediction of outcome from MRI and general movements assessment after hypoxic-ischaemic encephalopathy in low-income and middle-income countries: data from a randomised controlled trial
}

\author{
Karoline Aker 자 , ${ }^{1,2}$ Niranjan Thomas ${ }^{\circ}{ }_{1}{ }^{3}$ Lars Adde, ${ }^{1,4}$ Beena Koshy, ${ }^{5}$ \\ Miriam Martinez-Biarge, ${ }_{1}^{6}$ Ingeborg Nakken, ${ }^{7}$ Caroline S Padankatti, ${ }_{1}^{5}$ Ragnhild Støen ${ }^{1,2}$
}

\begin{abstract}
- Additional supplemental material is published online only. To view, please visit the journal online (http://dx.doi. org/10.1136/archdischild2020-321309)
\end{abstract}

For numbered affiliations see end of article.

\section{Correspondence to}

Dr Karoline Aker, Department of Clinical and Molecular Medicine, Norwegian University of Science and Technology, Faculty of Medicine and Health Sciences, 7491 Trondheim, Norway; karoline.aker@ntnu.no

Received 1 December 2020 Revised 26 March 2021 Accepted 20 April 2021
Check for updates

(C) Author(s) (or their employer(s)) 2021. Re-use permitted under CC BY-NC. No commercial re-use. See rights and permissions. Published by BMJ.

To cite: Aker K, Thomas N, Adde L, et al. Arch Dis Child Fetal Neonatal Ed Epub ahead of print: [please include Day Month Year]. doi:10.1136/

archdischild-2020-321309

\section{ABSTRACT}

Objective To evaluate the accuracy of neonatal MRI and general movements assessment (GMA) in predicting neurodevelopmental outcomes in infants with hypoxicischaemic encephalopathy (HIE).

Design Secondary analyses of a randomised controlled trial (RCT).

Setting Tertiary neonatal intensive care unit in India. Methods Fifty infants with HIE were included in an RCT of therapeutic hypothermia (25 cooled and 25 noncooled). All infants underwent brain MRI at day 5, GMA at 10-15 weeks and outcome assessments including Bayley Scales of Infant and Toddler Development, third edition, at 18 months. Associations between patterns of brain injury, presence/absence of fidgety movements (FMs) and outcomes were assessed.

Results Seventeen of 47 (36\%) had adverse outcome (5 (21\%) cooled vs 12 (52\%) non-cooled, $p=0.025)$. Eight infants died (four before an MRI, another three before GMA). Two developed severe cerebral palsy and seven had Bayley-III motor/cognitive composite score $<85$. Twelve $(26 \%)$ had moderately/severely abnormal MRI and nine (23\%) had absent FMs. The positive predictive value $(95 \% \mathrm{Cl})$ of an adverse outcome was $89 \%$ (53\% to $98 \%$ ) for moderate/severe basal ganglia and thalami (BGT) injury, 83\% (56\% to 95\%) for absent/ equivocal signal in the posterior limb of the internal capsule (PLIC) and 67\% (38\% to 87\%) for absent FMs. Negative predictive values $(95 \% \mathrm{Cl})$ were $85 \%$ (74\% to $92 \%$ ) for normal/mild BGT injury, $90 \%$ (78\% to $96 \%$ ) for normal PLIC and 86\% (74\% to 93\%) for present FMs.

Conclusion(s) Neonatal MRI and GMA predicted outcomes with high accuracy in infants with HIE. The GMA is a feasible low-cost method which can be used alone or complementary to MRI in low-resource settings to prognosticate and direct follow-up. Trial registration number CTRI/2013/05/003693.

\section{INTRODUCTION}

Perinatal asphyxia is ranked among the top 20 causes of death at any age globally. ${ }^{1}$ The main burden of disease is in low-income and middle-income countries (LMICs), where the incidence of hypoxicischaemic encephalopathy (HIE) is 10-20 times higher than in high-income countries (HICs). ${ }^{2}$ Therapeutic hypothermia (TH) has been found to

\section{What is already known on this topic?}

- The main burden of hypoxic-ischaemic encephalopathy is in low-income and middleincome countries, and many infants die or survive with disability.

- Neonatal MRI and general movements assessment are recommended assessments to predict cerebral palsy in infants at risk of perinatal brain injury.

- The literature on outcome and outcome prediction after hypoxic-ischaemic encephalopathy in low-income and middleincome countries is sparse.

\section{What this study adds?}

This study from South India showed similar patterns of brain injury in infants with hypoxicischaemic encephalopathy as trials from highincome countries.

- Both neonatal MRI and general movements assessment predicted neurodevelopmental outcome with high accuracy.

- Assessment of fidgety movements is a feasible low-cost method to prognosticate and direct follow-up in low-resource settings.

reduce mortality and major disability in survivors by $25 \%$ (relative risk) with a number needed to treat of $7 .^{4}$ The therapeutic hypothermia in India (THIN) study showed a neuroprotective effect of TH on early MRI biomarkers, ${ }^{5}$ but data on neurodevelopmental outcomes in LMICs are scarce.

Outcomes after HIE range from full recovery to death or survival with different degrees of disability. ${ }^{67}$ The prevalence and distribution of outcomes may differ between settings, and factors like maternal morbidities, nutritional status and infections may influence both the pattern of brain injury and the effect of cooling in infants with HIE. ${ }^{8-12}$ A study from South India reported more white matter (WM) injury in HIE infants as compared with studies from HICs. ${ }^{13}$

Prediction of outcomes in infants with HIE is essential for affected families, and important for 


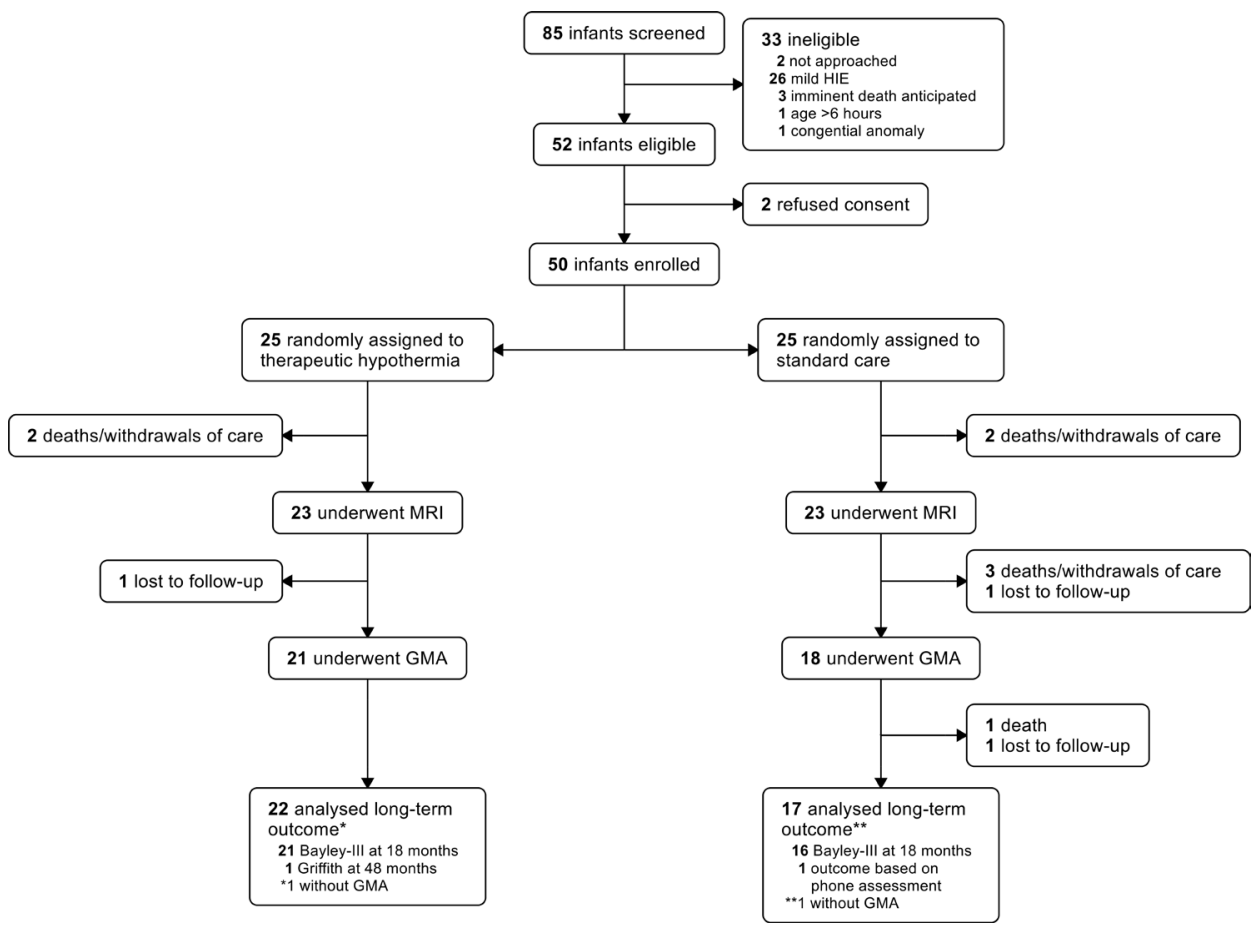

Figure 1 Trial profile. Bayley-III, Bayley Scales of Infant and Toddler Development (third edition); GMA, general movements assessment; Griffith, Griffith Mental Development Scales; HIE, hypoxic-ischaemic encephalopathy.

Table 1 Outcomes according to neonatal MRI*

\begin{tabular}{|c|c|c|c|c|}
\hline & $\begin{array}{l}\text { Normal/Mildly } \\
\text { abnormal MRI } \\
(\mathrm{n}=34)\end{array}$ & $\begin{array}{l}\text { Moderately/ } \\
\text { Severely } \\
\text { abnormal MRI } \\
(\mathrm{n}=12)\end{array}$ & $\begin{array}{l}\text { Total } \\
(n=46)\end{array}$ & $P$ value \\
\hline Fidgety movements & & & & $0.001 \dagger$ \\
\hline Absent (-) & $3 / 31(9.7)$ & $6 / 8(75.0)$ & 9/39 (23.1) & \\
\hline Sporadic (+/-) & $10 / 31(32.3)$ & 0 & $10 / 39(25.6)$ & \\
\hline Intermittent (+) & 16/31 (51.6) & $2 / 8(25.0)$ & 18/39 (46.2) & \\
\hline Continual (++) & $1 / 31(3.2)$ & 0 & 1/39 (2.6) & \\
\hline Exaggerated & 1/31 (3.2) & 0 & 1/39 (2.6) & \\
\hline Adverse outcome $\ddagger$ & $5 / 32(15.6)$ & 8/11 (72.7) & $13 / 43(30.2)$ & $<0.001$ \\
\hline Death & 0 & $4 / 12(33.3)$ & $4 / 46(8.7)$ & $<0.001$ \\
\hline $\begin{array}{l}\text { Bayley-III cognitive } \\
\text { composite score }\end{array}$ & & & & 0.001 \\
\hline$<70$ & 0 & $3 / 7(42.9)$ & $3 / 38(7.9)$ & \\
\hline $70-84$ & 4/31 (12.9) & 1/7 (14.3) & $5 / 38(13.2)$ & \\
\hline$\geq 85$ & $27 / 31(87.1)$ & $3 / 7$ (42.9) & $30 / 38$ (78.9) & \\
\hline $\begin{array}{l}\text { Bayley-III motor } \\
\text { composite score }\end{array}$ & & & & 0.002 \\
\hline$<70$ & 0 & $3 / 7$ (42.9) & $3 / 38$ (7.9) & \\
\hline $70-84$ & $3 / 31(9.7)$ & 0 & $3 / 38$ (7.9) & \\
\hline$\geq 85$ & 28/31 (90.3) & $4 / 7$ (57.1) & $32 / 38(84.2)$ & \\
\hline Cerebral palsy & 1/31 (3.2) & $3 / 7$ (42.9) & $4 / 38(10.5)$ & 0.002 \\
\hline \multicolumn{5}{|l|}{ GMFCS } \\
\hline Level 1 & 1/31 (3.2) & $1 / 7(14.3)$ & 2/38 (5.3) & \\
\hline Level 5 & 0 & $2 / 7(28.6)$ & 2/38 (5.3) & \\
\hline
\end{tabular}

Data are $\mathrm{n} / \mathrm{N}(\%)$. Denominators vary according to available data.

*Normal/Mildly abnormal MRI defined as normal/mild basal ganglia and thalami score and/ or normal/mild/moderate white matter score. Moderately/Severely abnormal MRI defined as moderate/severe basal ganglia and thalami score and/or absent posterior limb of the internal capsule and/or severe white matter score.

tP value based on Fisher's exact test on absent versus present FMs.

¥Adverse outcome defined as death, cerebral palsy with GMFCS level 3-5 or Bayley-III cognitive and/or motor composite score $<85$.

Bayley-III, Bayley Scales of Infant and Toddler Development (third edition); GMFCS, Gross Motor Function Classification System. clinicians to direct resources for follow-up. Neonatal MRI and the observation of spontaneous movements using the general movements assessment (GMA) are recommended tools to predict cerebral palsy (CP) in high-risk infants. ${ }^{14}$ Abnormal signal intensity in the basal ganglia and thalami (BGT) on MRI, which is the typical injury pattern after an acute hypoxic-ischaemic insult, is strongly associated with later motor impairment. ${ }^{15}$ On the other hand, predominant WM injury, carries a lower risk of motor impairment, but is more related to cognitive and behavioural impairments. ${ }^{16}$ Absence of fidgety movements (FMs) on GMA between 9 and 20 weeks post-term age is a strong marker for later CP. ${ }^{1718}$ In LMICs where resources are limited and MRI not easily available, GMA could be an ideal cost-effective predictive tool. However, most studies on GMA have been done in HICs and mainly in preterm populations.

The aim of this substudy is to evaluate the ability of early MR biomarkers and presence or absence of FMs to predict neurodevelopmental outcomes in infants with HIE included in a randomised controlled trial (RCT) on $\mathrm{TH}$ in India.

\section{METHODS}

\section{Study population}

This is a substudy of the THIN-study, a single-centre RCT of infants admitted with HIE to the neonatal intensive care unit (NICU) at the Christian Medical College Vellore, a tertiary care teaching hospital in rural south India. Approximately 15 000 babies are born at the hospital annually. The NICU has 75 beds and is a referral unit for a population of approximately 6 million. The unit offers level 3 neonatal intensive care including high-frequency oscillatory ventilation, inhaled nitric oxide and surgery. Fifty infants at or near term admitted before 5 hours after birth with signs of moderate/severe HIE were recruited between September 2013 and October 2015. The infants were randomly assigned to hypothermia with target core temperature $33.5^{\circ} \mathrm{C} \pm 0.5^{\circ} \mathrm{C}$ for 72 hours induced by a phase changing 


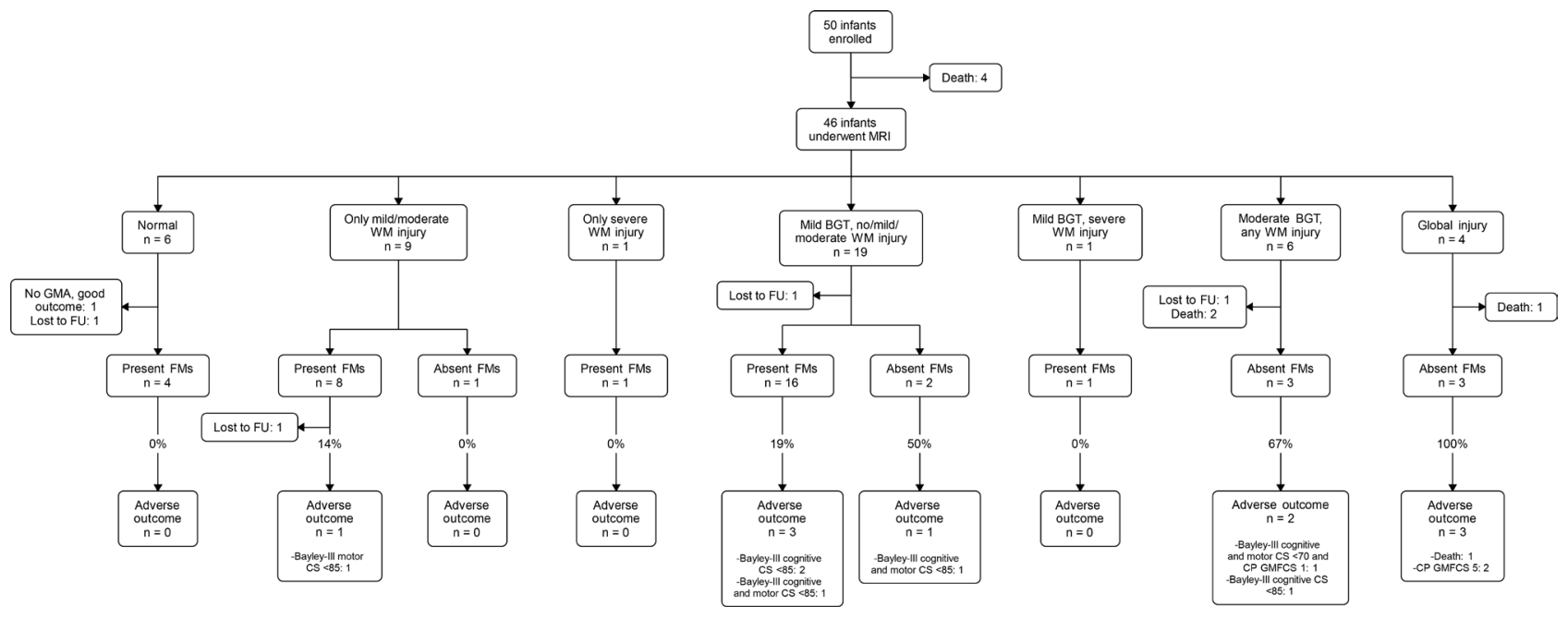

Figure 2 Flow chart showing patterns of brain injury, present or absent fidgety movements (FMs) and outcomes. BGT, basal ganglia and thalami; $\mathrm{CP}$, cerebral palsy; CS, composite score; FU, follow-up; GMA, general movements assessment; GMFCS, Gross Motor Function Classification System; WM, white matter.

material-based cooling device (MiraCradle Neonate Cooler, Pluss Advanced Technologies, India) or standard care with normothermia. Full description of the trial is published. ${ }^{5}$ Written parental consent was obtained after giving the parents an information leaflet and oral explanation.

\section{Early assessments}

All infants underwent cerebral MRI at $5 \pm 1$ days of age with a 3.0 T Philips Achieva scanner (Philips Healthcare, Best, The Netherlands; software V.3.2.3.1) using a standard protocol. ${ }^{5}$ The clinical images were reviewed by one neonatologist (MM-B) with experience in neonatal neuroimaging and scored in accordance with Rutherford et al. ${ }^{19}$ Images were classified as normal/ mildly abnormal (BGT score normal or mild and/or WM score normal, mild or moderate) or moderately/severely abnormal (moderate/severe BGT score and/or absent posterior limb of the internal capsule (PLIC), and/or severe WM score). ${ }^{19}$ Images were also categorised in seven patterns of brain injury: normal (no signs of abnormalities), only mildly/moderately abnormal WM, only severely abnormal WM, mildly abnormal BGT with normal or mildly/moderately abnormal WM, mildly abnormal BGT with severely abnormal WM, moderately abnormal BGT with any WM score and global brain injury (severely abnormal BGT, WM and cortex with absent PLIC and brainstem abnormalities).
At 10 to 15 weeks post-term age, a video for GMA was recorded using a standardised set-up with a digital camera (Sanyo VPC-HD2000, SANYO Electric, Osaka, Japan). The infants were filmed once in active wakefulness laying on a mattress in supine position. The video was analysed independently by two certified GMA observers in accordance with Prechtl's method. ${ }^{18}$ FMs were classified in accordance with their temporal organisation as continuous, intermittent or sporadic based on their presence and interspersed pauses. ${ }^{20}$ The category exaggerated was used if the FMs were exaggerated in speed and amplitude. FMs were defined as 'absent' if they were not observed, and 'present' if present in a continuous, intermittent, sporadic or exaggerated pattern. If disagreement occurred, the observers reassessed the video and reached consensus.

\section{Neurodevelopmental outcome}

At 18 months, an experienced developmental paediatrician and a clinical psychologist performed a complete neurological examination, the Bayley Scales of Infant and Toddler Development, third edition (Bayley-III), ${ }^{21}$ and clinical assessment of vision and hearing. CP and CP subtype were diagnosed in accordance with Surveillance of Cerebral Palsy in Europe ${ }^{22}$ and gross motor function was classified using the Gross Motor Function Classification System (GMFCS). ${ }^{23}$ Adverse outcome was defined as
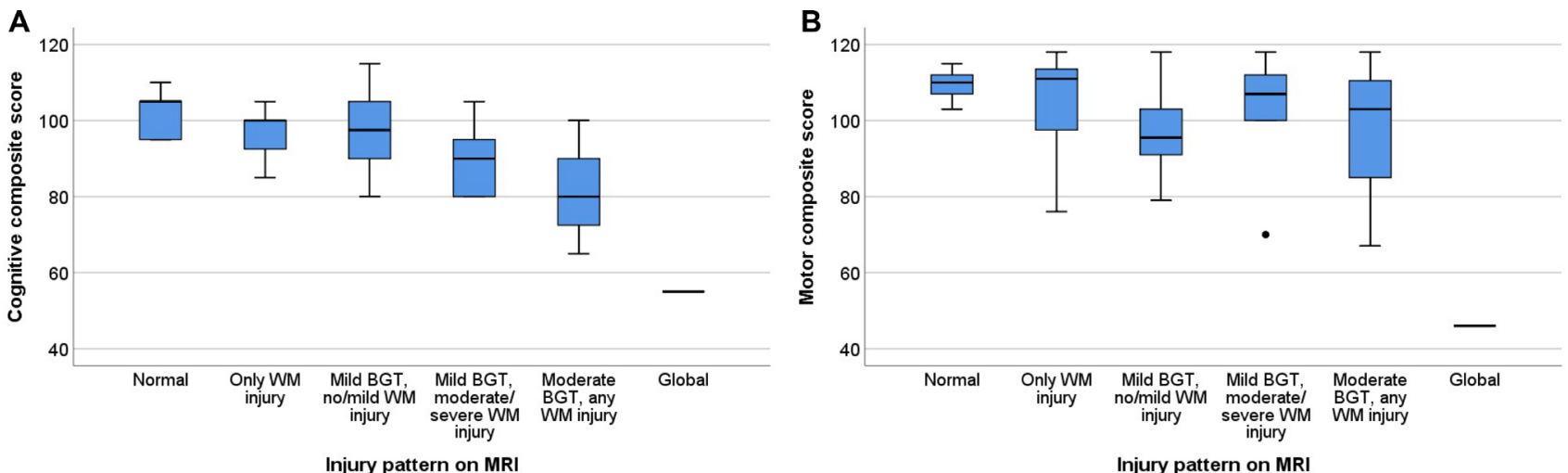

Figure 3 Bayley-III cognitive (A) and motor (B) composite score (median, first and third quartile and range) according to injury pattern on neonatal MRI. Bayley-III, Bayley Scales of Infant and Toddler Development, third edition; WM, white matter; BGT, basal ganglia and thalami. 
A

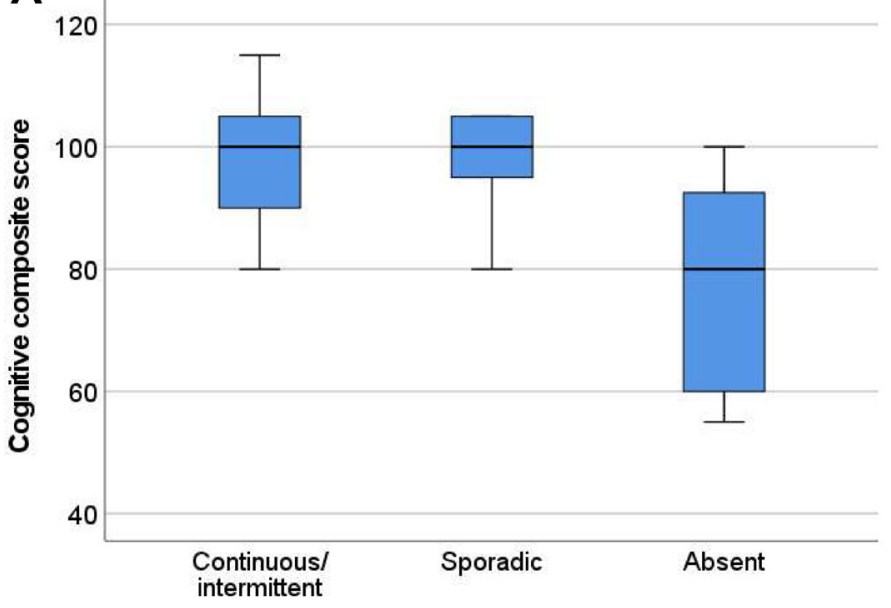

Temporal organisation of FMs
B

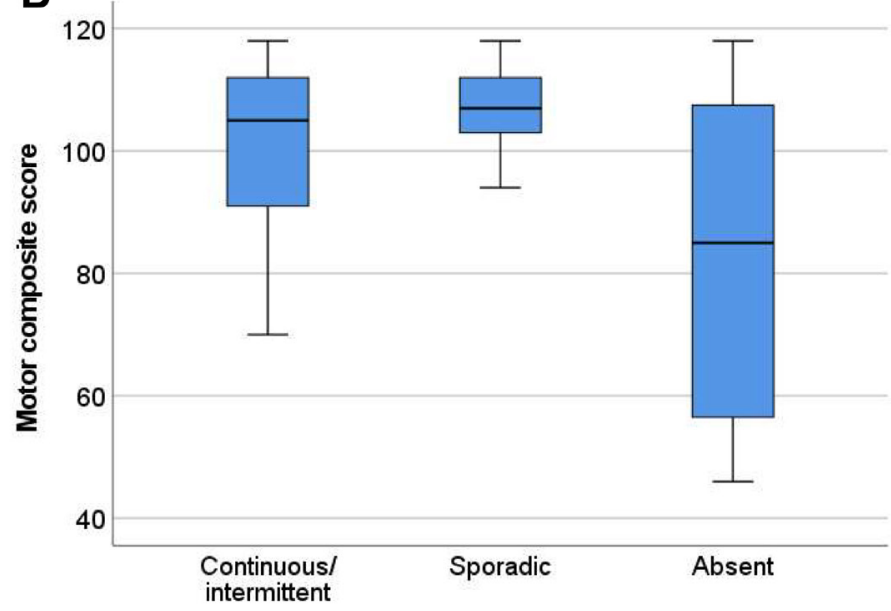

Temporal organisation of FMs

Figure 4 Bayley-III cognitive (A) and motor (B) composite score (median, first and third quartile and range) according to temporal organisation of fidgety movements (FMs) on general movements assessment. One infant with exaggerated FMs is not included in the box plot but had Bayley-III cognitive and motor composite score of 95 and 85, respectively. Bayley-III, Bayley Scales of Infant and Toddler Development, third edition.

a Bayley-III cognitive and/or motor composite score (CS) $<85$ $(-1 \mathrm{SD}),{ }^{24} 25$ a diagnosis of CP GMFCS level 3-5, impaired sensory/communication outcomes (blindness or deafness), ongoing seizure disorder or death related to associated causes. The Bayley-III language CS was not used in the analysis due to the infants' young age and the subjectivity of their non-English application. One infant had a normal Griffith Mental Development Scales (GMDS) assessment at 48 months and was assigned a Bayley motor and cognitive CS of 105 and 107, respectively, due to higher scores found in testing of typically developing children. ${ }^{2627}$ Another infant's outcome was based on phone assessment and classified as normal in the binary analysis of outcome.
All assessors of MRI, GMA and outcomes were blinded to the intervention and the results of former examinations.

\section{Statistics}

Data were analysed using SPSS V.25 (IBM, Chicago, Illinois, USA). Clinical characteristics and outcomes were summarised with absolute numbers (percentages) for categorical variables and means (SD) or medians (IQR) for continuous variables. Group differences in outcomes were analysed by $\chi^{2}$ tests, Fisher's exact tests, linear-by-linear associations, Student's t-tests or Mann-Whitney U tests, as appropriate. Sensitivity, specificity,

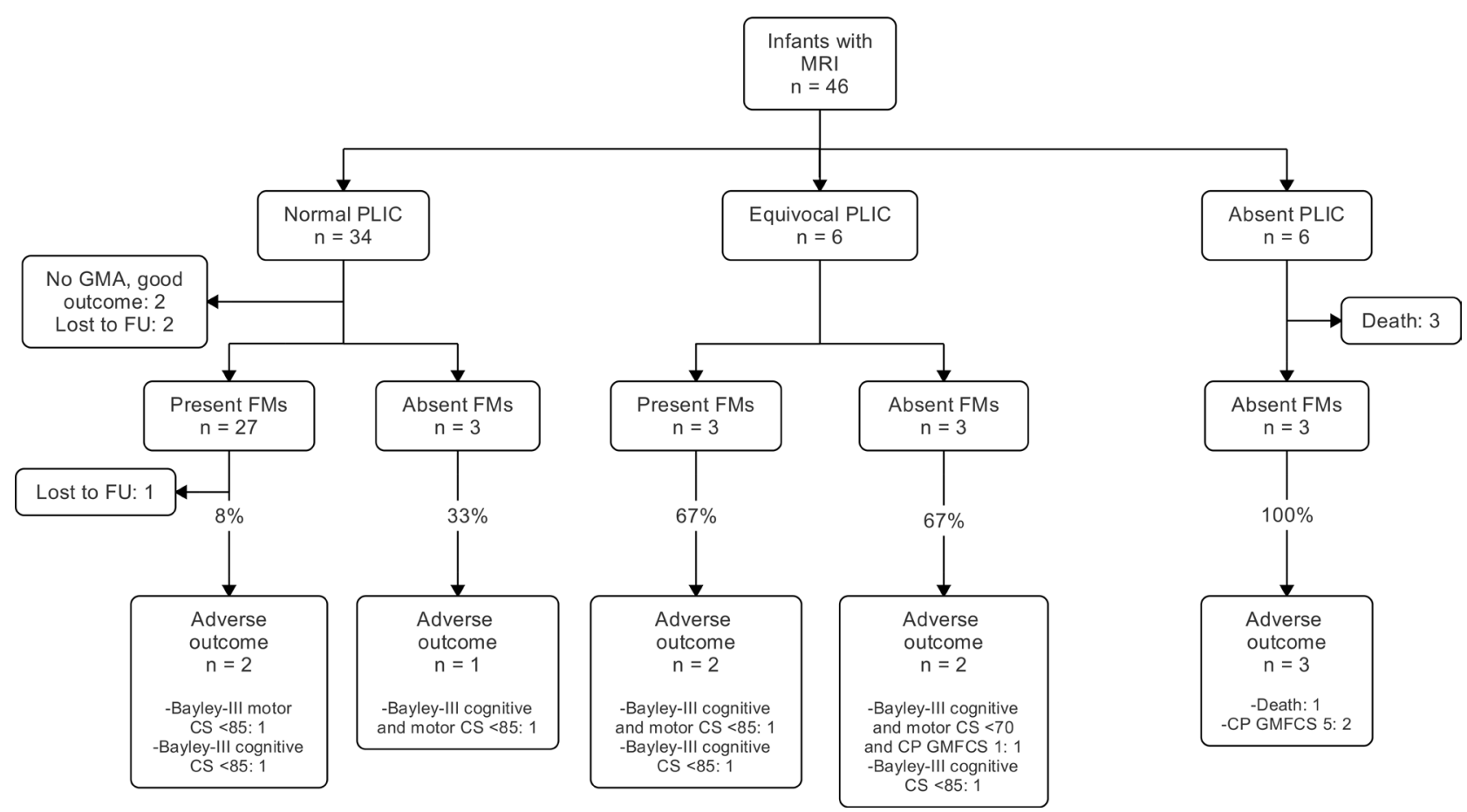

Figure 5 Flow chart showing involvement of posterior limb of internal capsule (PLIC), present or absent fidgety movements (FMs) and outcomes. CS, composite score; CP, cerebral palsy; FU, follow-up; GMA, general movements assessment; GMFCS, Gross Motor Function Classification System. 
Table 2 Predictive ability of MRI abnormalities and general movements assessment for adverse outcome*

\begin{tabular}{|c|c|c|c|c|c|}
\hline & Sensitivity & Specificity & PPV & NPV & Accuracy \\
\hline Moderately/Severely abnormal MRI† & 61.5 (31.6 to 86.1) & 90.0 (73.5 to 97.9$)$ & 72.7 (45.6 to 89.5$)$ & 84.4 (72.9 to 91.6$)$ & 81.4 (66.6 to 91.6$)$ \\
\hline Moderate/Severe BGT injury & 61.5 (31.6 to 86.1$)$ & 96.7 (82.8 to 99.9$)$ & 88.9 (52.6 to 98.3$)$ & 85.3 (74.4 to 92.1$)$ & 86.1 (72.1 to 94.7$)$ \\
\hline Absent PLIC & 46.2 (19.2 to 74.9$)$ & 100.0 (88.4 to 100.0$)$ & 100.0 & 81.1 (72.2 to 87.6$)$ & 83.7 (69.3 to 93.2$)$ \\
\hline Equivocal/Absent PLIC & $76.9(46.2$ to 95.0$)$ & 93.3 (77.9 to 99.2$)$ & 83.3 (55.9 to 95.2 ) & 90.3 (77.5 to 96.2$)$ & 88.4 (74.9 to 96.1$)$ \\
\hline Absent FMs & 60.0 (26.2 to 87.8$)$ & 89.3 (71.8 to 97.7$)$ & 66.7 (38.0 to 86.7 ) & 86.2 (74.3 to 93.1$)$ & 81.6 (65.7 to 92.3$)$ \\
\hline Sporadic or absent FMs & 70.0 (34.8 to 93.3 ) & 57.1 (37.2 to 75.5 ) & 36.8 (24.4 to 51.3$)$ & 84.2 (66.3 to 93.6$)$ & 60.5 (43.4 to 76.0 ) \\
\hline
\end{tabular}

Data are $\%(95 \% \mathrm{Cl})$.

*Adverse outcome defined as death, cerebral palsy with Gross Motor Function Classification System level 3-5 or Bayley-lll cognitive and/or motor composite score $<85$.

† Moderately/Severely abnormal MRI defined as moderate/severe BGT score and/or absent PLIC and/or severe WM score.

BGT, basal ganglia and thalami; FMs, fidgety movements; NPV, negative predictive value; PLIC, posterior limb of the internal capsule; PPV, positive predictive value.

positive predictive value (PPV), negative predictive value (NPV) and the predictive accuracy for adverse outcome were calculated for moderately/severely abnormal MRI, moderate/severe BGT injury, absent PLIC, combined absent and equivocal PLIC, absent FMs and combined absent and sporadic FMs. The CIs for the predictive values were calculated using a free online statistical calculator from MedCalc (MedCalc Software, Ostend, Belgium). ${ }^{28}$ There were no corrections made for multiple comparisons. Differences with $\mathrm{p}$ values $<0.05$ were considered statistically significant.

\section{RESULTS}

Fifty neonates were recruited, 25 in the cooled and 25 in the non-cooled group (figure 1). Demographics, neonatal characteristics and outcomes according to treatment group are shown in online supplemental eTable 1 and 2. Forty-six infants who survived until day 4 underwent MRI at a median age of 5 days (range 4-7), and $12(26 \%)$ of these had moderately/severely abnormal MRI (table 1). The most frequent pattern of brain injury was mildly abnormal BGT with normal or mildly/moderately abnormal WM (online supplemental eTable 1). A video recording for GMA was obtained in 39 infants at a median postterm age of 12 weeks (range 8-16), and 9 (23\%) infants had absent FMs. Two came for GMA before 10 weeks, and both had intermittent FMs. Assessment of outcome using Bayley-III was available in 37 infants at a median age of 18 months (range 16-21), and another infant had Bayley-III score estimated based on a normal assessment with GMDS. Three infants were lost to follow-up (one with completely normal MRI, one with moderate WM injury and present FMs and one with moderate BGT and severe WM injury).

Seventeen of 47 (36\%) infants with available follow-up data had adverse outcome, 5 cooled and 12 non-cooled (21\% vs 52\%, $\mathrm{p}=0.025)$. Eight infants died of reasons related to HIE, seven in the neonatal period and one at 12 months. The nine survivors with adverse outcomes included two with CP GMFCS level 5 (both had Bayley-III scores $<70$ ), and seven who had a Bayley-III motor and/or cognitive CS $<85$ (one with bilateral hearing loss, and one with CP GMFCS level 1 and Bayley-III scores $<70$ ).

\section{Prediction of outcome}

MRI and outcome

Adverse outcome was significantly more common among infants with moderately/severely abnormal than normal/mildly abnormal MRI ( 8 of 11 and 5 of 32, respectively; $\mathrm{p}<0.001$, table 1). The eight infants with moderately/severely abnormal MRI and adverse outcome had either a global brain injury pattern or moderate BGT injury with absent or equivocal PLIC (figure 2). All five infants with normal/mildly abnormal MRI and adverse outcome had either mild BGT injury with normal or equivocal PLIC and/or moderate WM injury (online supplemental eTable 3).

Bayley-III cognitive CS was significantly lower in infants with moderately/severely abnormal MRI compared with those with normal/mildly abnormal MRI (median (IQR) 80 (55-100) vs 95 (90-105), respectively, $\mathrm{p}=0.030)$. Infants with moderately/ severely abnormal MRI also had lower Bayley-III motor CS than infants with normal/mildly abnormal MRI, but this difference was not significant (median (IQR) 100 (46-115) vs 107 (91-112), $\mathrm{p}=0.335)$. The Bayley-III cognitive and motor CS according to injury patterns on MRI are shown in figure 3.

\section{GMA and outcome}

Six of nine (67\%) infants with absent FMs had adverse outcome: one died, two developed severe CP and three had Bayley-III motor and/or cognitive CS $<85$. The remaining three infants with absent FMs had normal outcome. Most infants with present FMs (25 of 29) had normal outcome, and the four with adverse outcome all had Bayley-III motor and/cognitive CS 70-85 and none of them developed CP. Ten (26\%) infants had sporadic FMs, of whom one had adverse outcome (Bayley-III cognitive CS $<85)$.

Infants with present FMs had significantly higher Bayley-III cognitive CS than those with absent FMs (median (IQR) 100 (91-105) vs 80 (58-94), respectively, $\mathrm{p}=0.001)$. The Bayley-III motor CS was also higher, although not significant, in infants with present versus absent FMs (median (IQR) 107 (94-112) vs 85 (51-110), $\mathrm{p}=0.091)$. The Bayley-III cognitive and motor CS for the different temporal classifications of FMs are shown in figure 4 .

\section{MRI, GMA and outcome}

Different patterns of brain injury in relation to GMA and outcome are shown in figure 2. All infants with a completely normal MRI and available follow-up assessments had present FMs and favourable outcome. One infant with mild BGT and WM injury had exaggerated FMs and was diagnosed with CP GMFCS level 1 (normal Bayley-III scores). All six infants with moderate BGT injury or global brain injury who came for GMA had absent FMs, and five of them had an adverse outcome (figure 2). All six infants with absent PLIC either died or survived with severe CP (figure 5). Sensitivity, specificity, PPV, NPV and accuracy of MRI and GMA for adverse outcomes are shown in table 2.

Four infants had either moderately/severely abnormal MRI or absent FMs, and a normal outcome (online supplemental etable4). Two had severe WM injury as predominant injury pattern, and the other two had absent FMs but a normal/ mildly abnormal MRI. In addition, one cooled infant with both moderate BGT injury, equivocal PLIC and absent FMs had a normal outcome. 


\section{DISCUSSION}

This substudy of an RCT on TH for HIE in a low-resource setting found that both neonatal MRI and GMA are closely associated with the combined outcome of death and adverse neurodevelopment at 18 months. The patterns of brain injury, with the majority of infants having BGT injury with or without WM injury, is similar to studies from high-income settings. ${ }^{19} 2930$ Normal/Mildly abnormal BGT, normal PLIC and present FMs were all highly predictive of a normal outcome in this study. The GMA at 10-15 weeks post-term age may be an alternative predictive tool in settings where MRI is not available.

The BGT injuries in our study were mainly mild. Severe BGT injury was less common compared with the National Institute of Child Health and Human Development (NICHD) trial and the Total Body Hypothermia (TOBY) trial, ${ }^{19}{ }^{30}$ and this is most likely due to the low proportion of infants with severe HIE in our study. We found a similar distribution of WM injury to the TOBY trial. ${ }^{19}$ Another Indian HIE trial reported predominantly WM injury and more than half of infants with moderate/severe HIE had normal BGT. ${ }^{13}$ WM injury is generally thought to be caused by more prolonged asphyxia or other causes of neonatal encephalopathy. ${ }^{16}$ Patterns of brain injury in HIE infants in different low-resource settings should be further explored.

Our results confirmed a close association between early spontaneous movements and neurodevelopmental outcome. Normal FMs at 2-3 months post-term age is a well-known predictor of a normal motor outcome. ${ }^{17} 31$ The association between FMs and cognitive outcome found in this study has also been reported by others. $^{32}{ }^{33}$ A normal outcome in 9 of 10 infants with sporadic FMs is in accordance with a large multicentre study where only $8 \%$ of infants with sporadic FMs developed CP. ${ }^{34}$

Moderate/Severe BGT abnormalities, equivocal/absent PLIC and absent FMs had the highest predictive accuracy for adverse outcome in this study. Ferrari et al found that the site and severity of brain injury on neonatal MRI and the results of GMA are closely correlated and has comparable accuracy in predicting motor outcome in non-cooled HIE infants. ${ }^{35}$ Similar predictive accuracy of MRI has been found in substudies of both the TOBY trial and the Infant Cooling Evaluation (ICE) trial. ${ }^{19}{ }^{36}$ Lally et $a l,{ }^{13}$ however, reported low sensitivity, but high specificity of MRI abnormalities in their study from South India, but only a small proportion of infants in that study had BGT injury.

In low-resource settings, MRI is often unavailable and expensive, while smartphones and internet access are cheap and available even in remote areas. ${ }^{37} \mathrm{~A}$ video recording for GMA can easily be performed using a simple video camera or even a smartphone. GMA is both time-efficient and cost-efficient and could be evaluated by remote assessment, which is especially important for LMICs. We suggest that in low-resource settings GMA is a good supplement, or even an alternative, to MRI to direct interventions and further follow-up in babies with HIE.

Although this study was not powered to detect differences in neurodevelopmental outcome between treatment groups, we found a reduction in adverse outcomes in cooled infants. Together with our previous report on reduction in several MRI biomarkers, we therefore state that TH for moderate HIE is feasible and may be neuroprotective in a low-resource setting. ${ }^{5}$ However, implementation of TH in LMICs should be limited to tertiary NICUs and requires development of guidelines and training programmes.

Limitations of this study include a small sample size and short duration of follow-up. This applies in particular to infants with severe WM injury and normal outcome, who are at risk of later cognitive/ behavioural disabilities. ${ }^{38}$ However, we had good follow-up rate.
Another limitation is the low proportion of infants with severe HIE, which is most likely due to selection bias.

\section{CONCLUSION}

In conclusion, we report that both neonatal MRI and GMA are strongly associated with neurodevelopmental outcome at 18 months in infants with HIE admitted to a tertiary care NICU in South India. GMA at 10-15 weeks post-term age has similar predictive accuracy as neonatal MRI and is therefore a feasible low-cost predictive tool in low-resource settings. The patterns of brain injury and incidence of adverse outcomes should be further explored in these settings.

\section{Author affiliations}

${ }^{1}$ Department of Clinical and Molecular Medicine, Norwegian University of Science and Technology, Faculty of Medicine and Health Sciences, Trondheim, Norway ${ }^{2}$ Department of Paediatrics, St. Olavs Hospital, Trondheim University Hospital, Trondheim, Norway

${ }^{3}$ Department of Neonatology, Christian Medical College Vellore, Vellore, Tamil Nadu, India

${ }^{4}$ Clinic of Clinical Services, St. Olavs Hospital, Trondheim University Hospital, Trondheim, Norway

${ }^{5}$ Department of Developmental Paediatrics, Christian Medical College Vellore, Vellore, Tamil Nadu, India

${ }^{6}$ Department of Paediatrics, Imperial College London, London, UK

${ }^{7}$ Norwegian Advisory Unit for Functional MRI, Department of Radiology, St. Olavs Hospital, Trondheim University Hospital, Trondheim, Norway

Acknowledgements The authors thank all the staff of the Department of Neonatology, the Christian Medical College (CMC), Vellore who took care of the study babies, and the research officers for adequate recruitment and collection of clinical data. The authors thank Live Eikenes (Department of Circulation and Medical Imaging, Norwegian University of Science and Technology (NTNU)) and Asta Håberg (Department of Neuromedicine and Movement Science, NTNU and Department of Radiology and Nuclear Medicine, St. Olavs Hospital, Trondheim University Hospital) for contribution in making the MRI protocol. The authors thank Toril Larsson Fjørtoft (Clinic of Clinical Services, St. Olavs Hospital, Trondheim University Hospital and Institute of Clinical and Molecular Medicine, NTNU) for participation in GMA analysis. The authors thank Associate Professor in Statistics Turid Follestad (Clinical Research Unit (entral Norway, NTNU) for statistical counselling. The authors also thank members of the Data Safety Monitoring Board at the CMC for monitoring the study.

Contributors NT was study PI. KA performed the literature search, conceptualised and designed the study, wrote the manuscript, performed the data analysis and data interpretation and reviewed and revised the manuscript. NT did the literature search, conceptualised and designed the study, enrolled patients, collected clinical data, was involved in data analysis and data interpretation and reviewed and revised the manuscript. LA did the GMA analysis, was involved in data analysis and data interpretation and reviewed and revised the manuscript. MM-B did the MRI analysis, was involved in data analysis and data interpretation and reviewed and revised the manuscript. IN made the MRI protocol, implemented and adapted the protocol on the local scanner and reviewed and revised the manuscript. BK and CSP did the outcome assessments, and reviewed and revised the manuscript. RS did the literature search, conceptualised and designed the study, was involved in data analysis and data interpretation and reviewed and revised the manuscript.

Funding The trial was funded by the Liaison Committee for Education, Research and Innovation in Central Norway, and the Joint Research Committee between St. Olavs Hospital, Trondheim University Hospital, and the Faculty of Medicine, NTNU, Trondheim, Norway.

Disclaimer The funders/sponsors had no role in the design and conduct of the study; collection, management, analysis and interpretation of the data; preparation, review or approval of the manuscript or decision to submit the manuscript for publication.

Competing interests NT has a patent 1796/DEL/2013 Life cradle device for inducing neonatal hypothermia issued.

Patient consent for publication Not required.

Ethics approval The study was approved by the Institutional Review Board at the Christian Medical College (number 2013/8223) and the Regional Committee for Medical and Health Research Ethics in central Norway (number 2013/2167).

Provenance and peer review Not commissioned; externally peer reviewed.

Data availability statement Data are available on reasonable request. Anonymised data (including data dictionaries) will be made available on request to 
researchers who provide a methodologically sound proposal for use in achieving the goals of the approved proposal.

Supplemental material This content has been supplied by the author(s). It has not been vetted by BMJ Publishing Group Limited (BMJ) and may not have been peer-reviewed. Any opinions or recommendations discussed are solely those of the author(s) and are not endorsed by BMJ. BMJ disclaims all liability and responsibility arising from any reliance placed on the content. Where the content includes any translated material, BMJ does not warrant the accuracy and reliability of the translations (including but not limited to local regulations, clinical guidelines, terminology, drug names and drug dosages), and is not responsible for any error and/or omissions arising from translation and adaptation or otherwise.

Open access This is an open access article distributed in accordance with the Creative Commons Attribution Non Commercial (CC BY-NC 4.0) license, which permits others to distribute, remix, adapt, build upon this work non-commercially, and license their derivative works on different terms, provided the original work is properly cited, appropriate credit is given, any changes made indicated, and the use is non-commercial. See: http://creativecommons.org/licenses/by-nc/4.0/.

\section{ORCID iDs}

Karoline Aker http://orcid.org/0000-0002-1046-6109

Niranjan Thomas http://orcid.org/0000-0002-1651-3201

\section{REFERENCES}

1 World Health Organization. Global health estimates 2016: deaths by cause, age, sex, by country and by region, 2000-2016. Geneva, 2018.

2 Lawn J, Shibuya K, Stein C. No Cry at birth: global estimates of intrapartum stillbirths and intrapartum-related neonatal deaths. Bull World Health Organ 2005;83:409-17.

3 Montaldo P, Pauliah SS, Lally PJ, et al. Cooling in a low-resource environment: lost in translation. Semin Fetal Neonatal Med 2015;20:72-9.

4 Jacobs SE, Berg M, Hunt R, et al. Cooling for newborns with hypoxic ischaemic encephalopathy. Cochrane Database Syst Rev 2013:CD003311.

5 Aker K, Støen R, Eikenes L, et al. Therapeutic hypothermia for neonatal hypoxicischaemic encephalopathy in India (thin study): a randomised controlled trial. Arch Dis Child Fetal Neonatal Ed 2020;105:405-11.

6 van de Riet JE, Vandenbussche FP, Le Cessie S, et al. Newborn assessment and longterm adverse outcome: a systematic review. Am J Obstet Gynecol 1999;180:1024-9.

7 Shankaran S, Laptook A. Challenge of conducting trials of neuroprotection in the asphyxiated term infant. Semin Perinatol 2003;27:320-32.

8 Zhao J, Chen Y, Xu Y, et al. Effect of intrauterine infection on brain development and injury. Int I Dev Neurosci 2013;31:543-9.

9 Wilkinson DJ, Thayyil S, Robertson NJ. Ethical and practical issues relating to the global use of therapeutic hypothermia for perinatal asphyxial encephalopathy. Arch Dis Child Fetal Neonatal Ed 2011;96:F75-8.

10 Jenkins DD, Lee T, Chiuzan C, et al. Altered circulating leukocytes and their chemokines in a clinical trial of therapeutic hypothermia for neonatal hypoxic ischemic encephalopathy*. Pediatr Crit Care Med 2013:14:786-95.

11 Jenster M, Bonifacio SL, Ruel T, et al. Maternal or neonatal infection: association with neonatal encephalopathy outcomes. Pediatr Res 2014;76:93-9.

12 Tann CJ, Webb EL, Lassman R, et al. Early childhood outcomes after neonatal encephalopathy in Uganda: a cohort study. EClinicalMedicine 2018:6:26-35.

13 Lally PJ, Price DL, Pauliah SS, et al. Neonatal encephalopathic cerebral injury in South India assessed by perinatal magnetic resonance biomarkers and early childhood neurodevelopmental outcome. PLoS One 2014:9:e87874.

14 Novak I, Morgan C, Adde L, et al. Early, accurate diagnosis and early intervention in cerebral palsy: advances in diagnosis and treatment. JAMA Pediatr 2017;171:897-907.

15 Martinez-Biarge M, Diez-Sebastian J, Rutherford MA, et al. Outcomes after central grey matter injury in term perinatal hypoxic-ischaemic encephalopathy. Early Hum Dev 2010;86:675-82.
16 Martinez-Biarge M, Bregant T, Wusthoff $\mathrm{CJ}$, et al. White matter and cortical injury in hypoxic-ischemic encephalopathy: antecedent factors and 2-year outcome. J Pediatr 2012;161:799-807.

17 Kwong AKL, Fitzgerald TL, Doyle LW, et al. Predictive validity of spontaneous early infant movement for later cerebral palsy: a systematic review. Dev Med Child Neurol 2018;60:480-9.

18 Einspieler C, Prechtl HFR, Bos AF. Prechtl's method on the qualitative assessment of general movements in preterm, term and young infants. London: Mac Keith Press, 2004.

19 Rutherford M, Ramenghi LA, Edwards AD, et al. Assessment of brain tissue injury after moderate hypothermia in neonates with hypoxic-ischaemic encephalopathy: a nested substudy of a randomised controlled trial. Lancet Neurol 2010;9:39-45.

20 Einspieler C, Peharz R, Marschik PB. Fidgety movements - tiny in appearance, but huge in impact. J Pediatr 2016;92:564-70.

21 Bayley N. Bayley scales of infant and toddler development, third edition. San Antonio: TX: Harcourt Assessment, Inc, 2006.

22 Surveillance of Cerebral Palsy in Europe. Surveillance of cerebral palsy in Europe: a collaboration of cerebral palsy surveys and registers. surveillance of cerebral palsy in Europe (SCPE). Dev Med Child Neurol 2000;42:816-24.

23 Palisano R, Rosenbaum P, Walter $\mathrm{S}$, et al. Development and reliability of a system to classify gross motor function in children with cerebral palsy. Dev Med Child Neurol 1997:39:214-23.

24 Johnson S, Moore T, Marlow N. Using the Bayley-III to assess neurodevelopmental delay: which cut-off should be used? Pediatr Res 2014;75:670-4.

25 Jary S, Whitelaw A, Walløe L, et al. Comparison of Bayley-2 and Bayley-3 scores at 18 months in term infants following neonatal encephalopathy and therapeutic hypothermia. Dev Med Child Neurol 2013;55:1053-9.

26 Anderson PJ, Burnett A. Assessing developmental delay in early childhood - concerns with the Bayley-III scales. Clin Neuropsychol 2017;31:371-81.

27 Anderson PJ, De Luca CR, Hutchinson E, et al. Underestimation of developmental delay by the new Bayley-III scale. Arch Pediatr Adolesc Med 2010;164:352-6.

28 MedCalc Software Ltd. Diagnostic test evaluation calculator Ostend, Belgium, 2020. Available: https://www.medcalc.org/calc/diagnostic_test.php

29 Okereafor A, Allsop J, Counsell SJ, et al. Patterns of brain injury in neonates exposed to perinatal sentinel events. Pediatrics 2008:121:906-14.

30 Shankaran S, Barnes PD, Hintz SR, et al. Brain injury following trial of hypothermia for neonatal hypoxic-ischaemic encephalopathy. Arch Dis Child Fetal Neonatal Ed 2012;97:F398-404.

31 Goyen T-A, Morgan C, Crowle C, et al. Sensitivity and specificity of general movements assessment for detecting cerebral palsy in an Australian context: 2-year outcomes. J Paediatr Child Health 2020;56:1414-8.

32 Barnes F, Graham L, Loganathan P, et al. General movement assessment predicts neuro-developmental outcome in very low birth weight infants at two years - a fiveyear observational study. Indian J Pediatr 2021;88:28-33.

33 Fjørtoft T, Grunewaldt KH, Løhaugen GCC, et al. Assessment of motor behaviour in high-risk-infants at 3 months predicts motor and cognitive outcomes in 10 years old children. Early Hum Dev 2013;89:787-93.

34 Støen R, Boswell L, de Regnier R-A, et al. The predictive accuracy of the general movement assessment for cerebral palsy: a prospective, observational study of high-risk infants in a clinical follow-up setting. J Clin Med 2019;8. doi:10.3390/ jcm8111790. [Epub ahead of print: 25 Oct 2019].

35 Ferrari F, Todeschini A, Guidotti I, et al. General movements in full-term infants with perinatal asphyxia are related to basal ganglia and thalamic lesions. J Pediatr 2011;158:904-11.

36 Cheong JLY, Coleman L, Hunt RW, et al. Prognostic utility of magnetic resonance imaging in neonatal hypoxic-ischemic encephalopathy: substudy of a randomized trial. Arch Pediatr Adolesc Med 2012;166:634-40.

37 International Telecommunication Union and United Nations Educational, Scientific and Cultural Organization. The state of broadband 2020. Geneva, 2020

38 Miller SP, Ramaswamy V, Michelson D, et al. Patterns of brain injury in term neonatal encephalopathy. J Pediatr 2005;146:453-60. 